be ensured that patient care is not compromised, and outcome measures remain stable over the course of the day.

\section{PTU-084 THE USE OF PANORAMIC CAPSULE ENDOSCOPY IN OBSCURE GASTROINTESTINAL BLEEDING}

Stefania Chetcuti Zammit*, Mark E McAlindon, Reena Sidhu. Sheffield Teaching Hospitals, Sheffield, UK

\subsection{6/gutjnl-2019-BSGAbstracts.443}

Introduction Up to $64 \%$ of patients with obscure gastrointestinal bleeding (OGIB) have lesions that can be reached with a standard gastroscope [1]. Positive findings outside the small bowel (SB) have been detected on axial small bowel capsule endoscopy (SBCE) (PillCam system, (Given Imaging) in patients with OGIB in up to $22 \%$ of patients in the stomach and $6 \%$ in the colon [2]. Our aim was to assess the diagnostic yield (DY) of panoramic SBCE (Capsocam) for the upper gastrointestinal tract, SB and colon in patients with OGIB.

Methods Patients at a tertiary centre who underwent Capsocam as part of the examination of OGIB following negative gastroduodenoscopy and colonoscopy or CT colonography were included. This study was carried out over a 6 year period. Findings on SBCE were recorded.

Results Forty-eight patients (37, 77.1\% overt, 11, 22.9\% occult OGIB) were included in this study. Thirty $(62.5 \%)$ were males. Mean age was $67.5 \pm 16.5$ years. OGIB was present for a mean of 19.9 SD \pm 92 months. Only one SBCE (9.1\%) was incomplete within the occult OGIB group $(p=0.229)$. The panoramic SBCE had a diagnostic yield of $37.5 \%$ (18 patients) in the SB. It also picked up additional findings in the stomach $(n=5,10.4 \%)$ and in the colon $(n=3$, $6.3 \%$ ). Some patients had more than one pathology (table 1).

\begin{tabular}{|c|c|c|c|}
\hline Findings & Stomach n (\%) & Small bowel n (\%) & Colon $\mathrm{n}(\%)$ \\
\hline Blood & $1(2.1)$ & $8(16.7)$ & $3(6.3)$ \\
\hline Erosions/gastritis & $3(6.3)$ & $3(6.3)$ & 0 \\
\hline Ulcers & $1(2.1)$ & $1(2.1)$ & 0 \\
\hline Angioectasias & 0 & $8(16.7)$ & $1(2.1 \%)$ \\
\hline ? tumour & 0 & $1(2.1)$ & 0 \\
\hline Diverticulum & 0 & $2(4.2)$ & 0 \\
\hline
\end{tabular}

Conclusions Panoramic SBCE can be useful in the detection of lesions in the stomach and colon missed during upper and lower gastrointestinal examinations. It can also be better in the detection of pathologies in overt OGIB than in occult OGIB.

\section{REFERENCES}

1. Zaman A, Katon RM: Push enteroscopy for obscure gastrointestinal bleeding yields a high incidence of proximal lesions within reach of a standard endoscope. Gastrointest Endosc 1998, 47:37-76.

2. Riccioni ME, Urgesi R, Cianci R, Marmo C, Galasso D, Costamagna G: Obscure recurrent gastrointestinal bleeding: a revealed mystery? Scand I Gastroenterol 2014, 49:102-026.

\section{PTU-085 VIRTUAL CLINICS OFFER AN EFFECTIVE AND SAFE ADDITION TO STANDARD OUT-PATIENT HEPATOLOGY SERVICES}

${ }^{1}$ Lynsey Corless*, ${ }^{2}$ Shaw Keith Lau, ${ }^{2}$ Alison Stainsby. ${ }^{1}$ Hull and East Yorkshire Hospitals, UK; ${ }^{2}$ Hull York Medical School, UK

\subsection{6/gutjnl-2019-BSGAbstracts.444}

Introduction Many patients with abnormal liver function tests/ steatosis do not have serious liver disease, and risk assessment for advanced disease can often be performed without face to face out-patient (OPC) review using laboratory results and other data. Virtual clinics (VC) may offer a useful adjunct to OPC increasing capacity for those requiring specialist care, and reduced patient visits. We piloted a consultant-led VC and assessed impact on OPC use.

Methods A retrospective audit was undertaken examining records of patients referred to liver VC/OPC (excluding viral hepatitis) in the year July 201-8. Clinical/demographic data were collected including referral detail, investigation results, and outcome of review. Appropriateness of referral route was reviewed by a consultant taking into account need for, and number of, OPC visits.

Results

VC 84 patients had initial review in VC. $72(85.7 \%)$ were community referrals, and $12(14.3 \%)$ from other specialties. There were no documented safety issues in patients managed in VC. 50 (59.5\%) were discharged after VC review, including $45(62.5 \%)$ of community referrals, equivalent to 4.2 unused OPC per month. Most were people with abnormal liver biochemistry or steatosis but low risk Fib4 score. Of 34 (40.5\%) who required subsequent OPC assessment 3 did not attend, $12(14.3 \%)$ required just 1 visit before discharge, $13(15.5 \%)$ needed - OPC, and $6(7.1 \%)$ entered long term follow up. In total $64.7 \%(22 / 34)$ of OPC need was solely for fibrosis assessment by Fibroscan, with 9/22 (40.9\%) discharged with normal result.

OPC OPC referrals were reviewed $(n=703)$, to determine how many would have been suitable for initial VC assessment. 456 first OPC attendances were identified after excluding cancellations/non-attenders. $40 \quad(8.7 \%)$ were discharged immediately, and $86(18.5 \%)$ were awaiting results to guide follow-up decision. These groups were considered potentially appropriate for VC, and reviewed in detail. Those placed on follow-up pathways were unlikely to be suitable and were not analysed further. Of those discharged at visit 1, 30 $(75 \%)$ would have been suitable for first review in VC, with 25 of those not requiring OPC attendance at all. Of 86 awaiting results, $58(67.4 \%)$ were suitable for first review in VC with 27 requiring no OPC input. A further 15 (17.4\%) could have avoided at least 1 visit. Taken together, this equates to $88 / 456(19.3 \%)$ visits which could have been conducted by VC, of whom 52/456 (11.4\%) required no OPC input at all. Including the 15 potentially avoidable visits, a total 67 OPC (5.6/month) could be saved $(9.5 \%$ of all OPC referrals).

Conclusions Most VC patients can be safely discharged without need for OPC, and expansion could further increase capacity for those requiring face-to-face specialist care. All our new referals are now initially vetted by VC. 